\section{Kidney \\ Blood Pressure Research}

\title{
Gene Expression Analysis in Tubule Interstitial Compartments Reveals Candidate Agents for IgA Nephropathy
}

\author{
Jinling Wang ${ }^{a} \quad$ Juan Cao \\ aDepartment of Nephrology; ${ }^{b}$ Department of Health Care, the Affiliated Hospital of Weifang Medical \\ University, Weifang 261031, Shandong Province, China
}

\author{
Key Words \\ IgA nephropathy $\bullet$ Gene ontology $\bullet$ Gene pairs $・$ Network $\bullet$ Small molecules
}

\begin{abstract}
Background/Aims: Our aim was to explore the molecular mechanism underlying development of IgA nephropathy and discover candidate agents for IgA nephropathy. Methods: The differentially expressed genes (DEGs) between patients with IgA nephropathy and normal controls were identified by the data of GSE35488 downloaded from GEO (Gene Expression Omnibus) database. The co-expressed gene pairs among DEGs were screened to construct the gene-gene interaction network. Gene Ontology (GO) enrichment analysis was performed to analyze the functions of DEGs. The biologically active small molecules capable of targeting IgA nephropathy were identified using the Connectivity Map (cMap) database. Results: A total of 55 genes involved in response to organic substance, transcription factor activity and response to steroid hormone stimulus were identified to be differentially expressed in IgA nephropathy patients compared to healthy individuals. A network with 45 co-expressed gene pairs was constructed. DEGs in the network were significantly enriched in response to organic substance. Additionally, a group of small molecules were identified, such as doxorubicin and thapsigargin. Conclusion: Our work provided a systematic insight in understanding the mechanism of IgA nephropathy. Small molecules such as thapsigargin might be potential candidate agents for the treatment of IgA nephropathy.
\end{abstract}

Copyright $@ 2014$ S. Karger AG, Basel

\section{Introduction}

IgA nephropathy is a common form of glomeruli inflammation, which is characterized by the deposition of glomerular IgA antibody in kidney accompanied with a heterogeneous clinical course [1]. The presence of glomerular IgA deposits often resulted in various diseases 


\section{Kidney Blood Pressure Research}

including dermatitis herpetiformis, celiac disease and systemic or immunologic disorders (systemic lupus erythematosus; cryoimmunoglobulinemia) [2]. Although IgA nephropathy is considered to be benign in early stage of patients with primary glomerular disease, many cases progress to later stage of renal disease resulting from IgA nephropathy [3, 4]. The incidence of primary IgA nephropathy was reported to be the highest in Asia [2] and there was an increasing trend for the prevalence of IgA nephropathy in United States [5]. IgA nephropathy has been a healthy concern throughout the world.

Numerous studies have made contributions to explore the mechanism and therapeutic method for IgA nephropathy, since it was first documented in 1968 [6]. The best option for treating the end-stage renal disease is renal transplantation. Although the survival of IgA nephropathy patients by renal transplantation is excellent [7], the recurrence is common in patients (20-60\%) which results in the renal function failure and graft loss [8]. Therapies in preserving renal functions and preventing substantial progress are necessary for patients with IgA nephropathy. The agents capable of reversing IgA nephropathy are mainly angiotensin-converting-enzyme inhibitors, corticosteroids, and n-3 polyunsaturated fatty acids. But there is no clear evidence that among the 3 classifications of drugs which is the better treatment [2]. In recent years, it is reported that mycophenolate mofetil (MMF) combined with prednisone showed advantage in the remission of severe IgA nephropathy [9]. However, there is no promising therapy available to treat the patients with IgA nephropathy by now.

In the present study, we utilized the biological informatics methods to assess the gene expression profiles derived from patients with IgA nephropathy and normal subjects. We aimed to potential mechanism underlying IgA nephropathy and discover the candidate effective agents for patients with IgA nephropathy.

\section{Materials and Methods}

\section{Affymetrix microarray data}

The microarray data of GSE35488 deposited by Heather N. Reich et al [10] was downloaded from GEO (Gene Expression Omnibus) database (http://www.ncbi.nlm.nih.gov/geo/). Total 31 specimens were used for the development of the gene expression profiles, which derived from tubule interstitial compartments of patients with IgA nephropathy $(n=25)$ and normal living donors $(n=6)$. The eligible patients including 18 males and 7 females aged from 19 to 84 years old (mean=36). Subjects with IgA nephropathy had a wide range in proteinuria (trace to $10 \mathrm{~g} /$ day) and renal function (normal to stage $4 / 5$ chronic kidney disease) [10]. The raw data and the annotation files were downloaded for further study, based on the platform of GPL96 (Affymetrix Human Genome U133A Array).

Data preprocessing and differential expression genes analysis

All the array data was preprocessed using Robust Multichip Average (RMA) method [11]. Normalization was performed at probe level. If multiple probes corresponding to the same gene, the mean value was calculated as the gene expression value for this gene. The differentially expressed genes (DEGs) in IgA nephropathy patients were analyzed by $\mathrm{R}$ multtest package [12]with reference to the data from normal subjects. The multiple testing correction was carried out to control the false discovery rate (FDR) with the application of BH (Benjamini-Hochberg) procedure [13]. Fold change (FC) of the expression of individual gene was also analyzed for DEGs analysis. DEGs with FDR $<0.05$ and $|\log F C|>1$ were considered to be significant.

Gene-annotation enrichment analysis

Gene Ontology (GO) database is a collection of a large number of gene annotation terms. DAVID (The Database for Annotation, Visualization and Integrated Discovery) Gene Functional Classification Tool has been developed for relating the functional terms with gene lists by clustering algorithm. We performed GO enrichment analysis of the DEGs using the DAVID functional classification tool. The multiple testing correction were carried out and FDR $<0.05$ was defined as the cutoff value. 


\section{Kidney Blood Pressure Research}

Wang/Cao: Candidate Agents for IgA Nephropathy

Co-expressed gene pairs and gene-gene interaction network construction

The genes with similar expression profiles are considered to have the common regulatory function and high degree of correlation [14]. R/EBcoexpress package applies empirical Bayesian approach to examine the correlations of gene pairs [15].

In this paper, we identified the co-expressed gene pairs using R/ EBcoexpress in $\mathrm{R}$ statistical programming language with the changes at mean expression level. The gene pairs were displayed with a co-expression value. The co-expression value $>0.6$ was defined as the cutoff value. Then the gene-gene interaction network with significant gene pairs was visualized using Cytoscape software [16]. The function annotation for genes in the network was performed with BiNGO (Biological Networks Gene Ontology tool) [17]. The multiple testing correction was performed to adjust p values according to $\mathrm{BH}$ procedure. FDR (adj p value) $<0.01$ was set as the threshold value.

\section{Identification of candidate small molecules}

Connectivity Map (cMap) database collects the gene expression profiles from cultured human cells treated with small molecules. cMap is widely used to identify small molecules sharing the mechanism of disease and drugs [18].

The gene set enrichment analysis (GSEA) was performed for the up-regulated DEGs and downregulated DEGs compared with the data from cMap database. The query small molecules were output with a connectivity score from +1 to -1 . The higher negative score indicated that the query small molecules suppressed disease, while the higher positive score revealed that the corresponding molecules induced the disease. A connectivity score nearer to " 0 " indicated no effect from query small molecules on disease. A $\mathrm{p}$ value for enrichment was also displayed. Candidate agents were identified with $\mathrm{p}$ value $<0.05$ and |connectivity score $\mid>0.9$.

\section{Results}

\section{Identification of differential expression genes}

After data preprocessing, we obtained 12001 probes of 12029 probes in raw gene expression profiles. Compared with the expression profiles from normal subjects, total of 55 genes were assessed to be differentially expressed in IgA nephropathy patients, among which, 3 genes were up-regulated and 52 ones were down-regulated. The DEGs with FDR $<0.05$ and $|\log \mathrm{FC}|>1$ were listed in Table 1. 


\section{Kidney Blood Pressure Research}

\begin{tabular}{l|l}
\hline Kidney Blood Press Res 2014;39:361-368 \\
\hline DOI: 10.1159/000355814 & $\begin{array}{l}\text { C 2014 S. Karger AG, Basel } \\
\text { www.karger.com/kbr }\end{array}$ \\
\hline Publisned onlIne: September 29, 2014 &
\end{tabular}

Wang/Cao: Candidate Agents for IgA Nephropathy

Function analysis Table 2. Function analysis of the differentially expressed gene sets

for DEGs

To analysis the

DEGs in the function level, we performed GO enrichment analysis for the DEGs. With FDR $<0.05$, all the DEGs were classified into 12 categories such as transcription factor activity, response to steroid hormone stimulus, doub-

\begin{tabular}{llcc}
\hline Term & \multicolumn{1}{c}{ Function } & Count & FDR \\
\hline GO:0010033 & response to organic substance & 17 & $9.43 \mathrm{E}-06$ \\
GO:0003700 & transcription factor activity & 16 & $4.29 \mathrm{E}-04$ \\
G0:0048545 & response to steroid hormone stimulus & 9 & $9.64 \mathrm{E}-04$ \\
GO:0003690 & double-stranded DNA binding & 7 & 0.00101 \\
GO:0009991 & response to extracellular stimulus & 9 & 0.00269 \\
G0:0043566 & structure-specific DNA binding & 7 & 0.01052 \\
G0:0009725 & response to hormone stimulus & 10 & 0.01454 \\
G0:0031667 & response to nutrient levels & 8 & 0.01473 \\
G0:0032496 & response to lipopolysaccharide & 6 & 0.01697 \\
G0:0002237 & response to molecule of bacterial origin & 6 & 0.02914 \\
GO:0008201 & heparin binding & 6 & 0.02926 \\
G0:0009719 & response to endogenous stimulus & 10 & 0.03191 \\
\hline
\end{tabular}

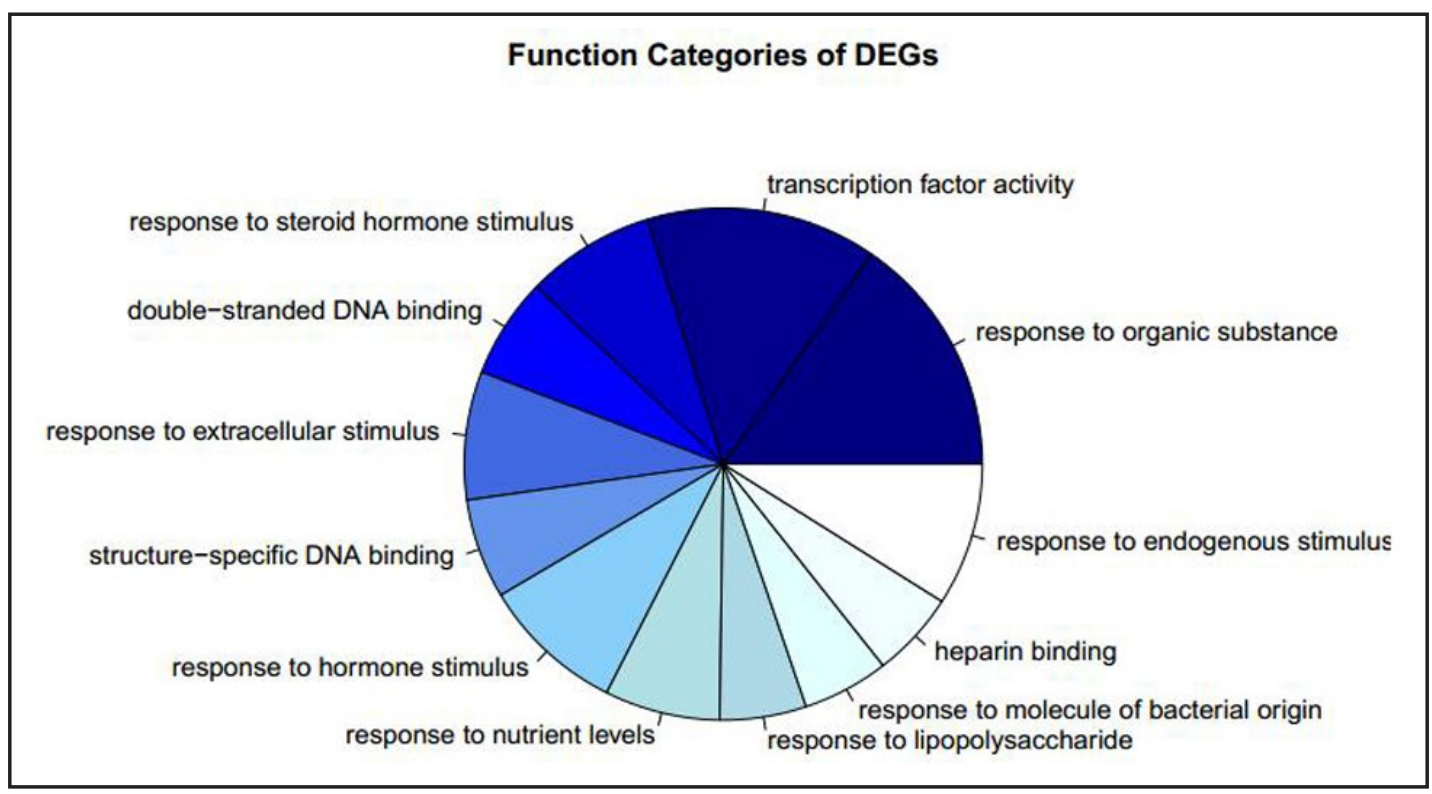

Fig. 1. Function categories of DEGs. The significance of the function categories declines from dark blue to white. DEG: differentially expressed genes.

le-stranded DNA binding, response to hormone stimulus and response to endogenous stimulus (Figure 1, Table 2). The most enriched GO term was reveled to be response to organic substance (Table 2).

Co-expressed gene pairs and gene-gene interaction network

In order to investigate the interactions of DEGs, we performed co-expressed gene pairs analysis. Total 45 gene pairs with co-expression value $>0.6$ were identified to be significant. With the application of Cytoscape, the gene-gene interaction network was established with the 45 co-expressed gene pairs (Figure 2).Then the functions of the genes in the network were further analyzed. As shown in Table 3, the significant function of the co-expression gene network was response to organic substance. Other functions included response to chemical stimulus, response to stress, organ development and system development.

Identification of candidate active small molecules

In order to identify the candidate agent for patients with IgA nephropathy, we applied the information of cMap database and verified with $\mathrm{p}$ value and enrichment scores. Total 


\section{Kidney \\ Blood Pressure Research}

of 5 potential small molecules were obtained with $\mathrm{p}<0.05$ and |connectivity score $>0.9$, including doxorubicin, atovaquone, mitonantrine, podophyllotoxin and thapsigargin. As shown in Table 4, the candidate small molecules of doxorubicin (connectivity score $=0.991$ ) and thapsigargin (connectivity score $=-0.966$ ) had highest positive score and negative score, respectively.

\section{Discussion}

IgA nephropathy is generally recognized as the most common form of glomerulonephritis worldwide $[19,20]$. IgA nephropathy is always accompanied with other numerous diseases which increased the risk of death. Although a large number of studies have been conducted to explore the pathogenesis of IgA nephropathy, the mechanism underlying IgA nephropathy progression has not been fully elucidated. The development of biological informatics approach for gene expression profiles advanced our ability to study human diseases [21, 22]. Accor-

dingly, the purpose of our study was to explore the potential mechanism underlying IgA nephropathy.

Our results showed that 55 genes were identified to be differentially expressed in tubule cells between IgA nephropathy patients and normal ones. GO analysis revealed that several function items were statistically over-represented by the DEGs, which mainly related with diverse biological process such as response to the up-regulated gene. \begin{tabular}{l|l}
\hline DOI: $10.1159 / 000355814$ & (C) 2014 S. Karger AG, Basel
\end{tabular}

Wang/Cao: Candidate Agents for IgA Nephropathy

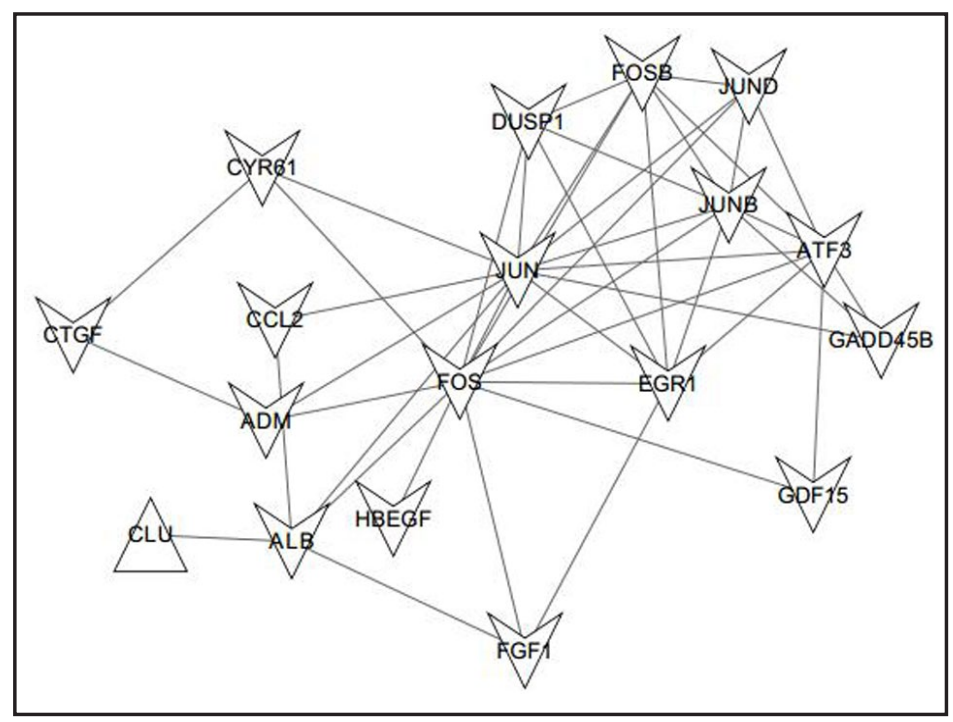

Fig. 2. Network of gene interaction pairs. The downward triangle represents downl-regulated gene, while the upward triangle represents

Table 3. Function annotation of genes in network

\begin{tabular}{lccl}
\hline GO-ID & $\begin{array}{c}\text { corr } \\
\text { p-value }\end{array}$ & x & Description \\
\hline 10033 & $3.52 \mathrm{E}-06$ & 10 & response to organic substance \\
42221 & $8.18 \mathrm{E}-05$ & 10 & response to chemical stimulus \\
6950 & $2.97 \mathrm{E}-04$ & 10 & response to stress \\
48513 & $3.02 \mathrm{E}-04$ & 10 & organ development \\
48731 & $1.09 \mathrm{E}-04$ & 12 & system development \\
48856 & $2.27 \mathrm{E}-04$ & 12 & anatomical structure development \\
48522 & $8.54 \mathrm{E}-07$ & 14 & positive regulation of cellular process \\
48518 & $1.79 \mathrm{E}-06$ & 14 & positive regulation of biological process \\
7275 & $2.31 \mathrm{E}-05$ & 14 & multicellular organismal development \\
32502 & $4.13 \mathrm{E}-05$ & 14 & developmental process \\
50896 & $1.31 \mathrm{E}-04$ & 14 & response to stimulus \\
32501 & $7.41 \mathrm{E}-04$ & 14 & multicellular organismal process \\
50794 & $2.31 \mathrm{E}-05$ & 18 & regulation of cellular process \\
50789 & $3.52 \mathrm{E}-05$ & 18 & regulation of biological process \\
65007 & $7.75 \mathrm{E}-05$ & 18 & biological regulation \\
\hline
\end{tabular}

Table 4. Candidate small molecules

\begin{tabular}{lcc}
\hline cMap name & Score & P value \\
\hline doxorubicin & 0.991 & 0.00002 \\
atovaquone & 0.949 & 0.00016 \\
mitoxantrone & 0.91 & 0.00154 \\
podophyllotoxin & -0.911 & 0.0001 \\
thapsigargin & -0.966 & 0.0001 \\
\hline
\end{tabular}

organic substance, transcription factor activity, response to steroid hormone stimulus and response to extracellular stimulus (Table 2). In order to better understand the interactions of disturbed genes, we established the network with significant gene pairs. The function analysis indicated response to organic substance was the most significant function of the network, which was corresponding to the results of GO analysis. 


\section{Kidney Blood Pressure Research}

There is a hypothesis that the exposure to organic solvents may be close associated with the pathogenesis of glomerulonephritis [23]. Previous evidence showed that subjects with occupational solvent exposure to paint, glue and styrene had higher risk for chronic glomerulonephritis compared with the ones with non-occupational exposure to solvent [24]. Recently, cigarette smoking was also proved to be a risk factor for chronic kidney disease in IgA nephropathy [25]. Nicotine, an organic compound in cigarette smoke was implied to modulate the endothelial dysfunction and accelerate the progression of kidney disease. Potent evidence showed that human mesangial cells possess the neuronal nicotinic acetylcholine receptors (nAChRs) [26]. The expression of nAChRs subunits promotes the nicotine effect on activating pro-fibrotic pathways, reducing glomerular filtration rate and glomerular filtration rate [27]. Accordingly, response to organic substance is a significant biological process in the development of kidney disease. Similarly, other clustered function by GEGs in this work may also play key roles in IgA nephropathy. Getting knowledge of the functions of disturbed genes is a better way to understand the pathogenesis.

Another aim of our study was to uncover the candidate small molecules that play key roles in IgA nephropathy development. Doxorubicin and thapsigargin were identified to be the significant small molecules in the development of IgA nephropathy. Doxorubicin, also named as adriamycin, is an anthracycline class of antibiotic and has been primarily used in the treatment of ovarian cancer [28] and others such as bladder cancer [29], breast cancer [30] and lung cancer [31]. Adriamycin is used restrictedly in clinical application for its toxicity which may result in cardiomyopathy and nephropathy development [32, 33]. Ariamycin can induce the development of hyperlipidaemia, glomerulosclerosis and tubulointerstitial fibrosis in normal subjects [34]. So it is widely used to construct nephropathy models in rats and mice. Although the mechanism of ariamycin induced nephropathy is far from being clear, ariamycin shows close association with IgA nephropathy development.

Furthermore, thapsigargin is served as non-competitive inhibitor of a class of enzymes such as endoplasmic reticulum (ER) $\mathrm{Ca}^{2+}$ ATPase [35] and is a tumor promoter in mammalian cells [36]. Tumorigenesis initiated by acute responses in cells. Thapsigargin induced the release of intracellular stored $\mathrm{Ca}^{2+}$ and rapidly increased the cytosolic free $\mathrm{Ca}^{2+}$ concentration by the potent inhibition of ER $\mathrm{Ca}^{2+}$ ATPase [37]. It is also reported that thapsigargin induces autophagy arrest and in turn increases the ER stress which ultimately leads to cell death. As outlined in recent studies, thapsigargin is available in experimental examining related with ER stress [38]. The clinical application of thapsigargin is mentioned in posterior capsule opacification treatment [39]. However, the evidence concerning the drug application of thapsigargin in IgA nephropathy is rare. Our work showed that thapsigargin had the highest negative score (connectivity score=-0.966) (Table 4), implying the capable of reversing disease. Therefore, attentions on the drug potential of thapsigargin may open vistas for developing novel therapy for IgA nephropathy treatment.

Although bioinformatics technologies have the potential to indentify and validate the candidate agents for serious diseases, many challenges still remain in this study. First, we downloaded the microarray data from GEO database instead of that developed by our research team. Second, for the specially designed platform of the microarray, we only obtained the array data from small number of samples. Besides, the results obtained in our paper were not validated by further experimental studies. Therefore, a large number of studies should be conducted to confirm the results in the future.

\section{Conclusion}

In summary, the biological informatics methods provide a systematic perspective to understand the progression of IgA nephropathy. The dysfunctions of significant gene sets may contribute to the development of IgA nephropathy, such as response to organic substance. Thapsigargin may be candidate agents for improving therapeutic effect on IgA nephropathy. The identification of active small molecules opened a novel insight to discover 


\section{Kidney \\ Blood Pressure Research}

the candidate agents for IgA nephropathy. However, the clinical applications of the candidate agents warrant further considerations.

\section{Disclosure Statement}

The authors of this manuscript state that they do not have any conflict of interests and nothing to disclose.

\section{Reference}

1 Radford MG, Donadio JV, Bergstralh EJ, Grande JP: Predicting renal outcome in IgA nephropathy. J Am Soc Nephrol 1997;8:199-207.

-2 Donadio JV, Grande JP: IgA nephropathy. N Engl J Med 2002;347:738-748.

-3 Maisonneuve P, Agodoa L, Gellert R, Stewart JH, Buccianti G, Lowenfels AB, Wolfe RA, Jones E, Disney AP, Briggs D: Distribution of primary renal diseases leading to end-stage renal failure in the United States, Europe, and Australia/New Zealand: results from an international comparative study. Am J Kidney Dis 2000;35:157-165.

4 Briganti EM, Dowling J, Finlay M, Hill PA, Jones CL, Kincaid-Smith PS, Sinclair R, Mcneil JJ, Atkins RC: The incidence of biopsy-proven glomerulonephritis in Australia. Nephrol Dial Transplant 2001;16:1364-1367.

5 Wyatt RJ, Julian BA, Baehler RW, Stafford CC, Mcmorrow RG, Ferguson T, Jackson E, Woodford SY, Miller PM, Kritchevsky S: Epidemiology of IgA nephropathy in central and eastern Kentucky for the period 1975 through 1994. Central Kentucky Region of the Southeastern United States IgA Nephropathy DATABANK Project. J Am Soc Nephrol 1998;9:853-858.

6 Berger J HN: Les depots intercapillaires d'IgA-IgG. Scand J Urol Nephrol 1968;74:594-695.

7 Ponticelli C, Traversi L, Feliciani A, Cesana BM, Banfi G, Tarantino A: Kidney transplantation in patients with IgA mesangial glomerulonephritis. Kidney Int 2001;60:1948-1954.

-8 Donadio JV, Jr., Grande JP: Immunoglobulin A nephropathy: a clinical perspective. J Am Soc Nephrol 1997;8:1324-1332.

-9 Liu X, Dewei D, Sun S, Xu G, Liu H, He L, Zhang P: Treatment of severe IgA nephropathy: mycophenolate mofetil/prednisone compared to cyclophosphamide/prednisone. Int J Clin Pharmacol Ther 2014;52:95102.

-10 Reich HN, Tritchler D, Cattran DC, Herzenberg AM, Eichinger F, Boucherot A, Henger A, Berthier CC, Nair V, Cohen CD: A molecular signature of proteinuria in glomerulonephritis. PLoS ONE 2010;5:e13451.

-11 Bolstad BM, Irizarry RA, Strand M, Speed TP: A comparison of normalization methods for high density oligonucleotide array data based on variance and bias. Bioinformatics 2003;19:185-193.

12 Gentleman R, Carey V, Huber W, Irizarry R, Dudoit S: Bioinformatics and computational biology solutions using R and Bioconductor. 2005, Vol. 746718470, Springer-Verlag, New York.

13 Benjamini Y, Hochberg Y: Controlling the false discovery rate: a practical and powerful approach to multiple testing. Series B (Methodological). J Royal Statistical Soc 1995:57:289-300.

14 Janaki C, Joshi RR: Motif detection in Arabidopsis: Correlation with gene expression data. In Silico Biol 2004;4:149-161.

15 Dawson JA, Ye S, Kendziorski C: R/EBcoexpress: an empirical Bayesian framework for discovering differential co-expression. Bioinformatics 2012;28:1939-1940.

16 Shannon P, Markiel A, Ozier O, Baliga NS, Wang JT, Ramage D, Amin N, Schwikowski B, Ideker T: Cytoscape: a software environment for integrated models of biomolecular interaction networks. Genome Res 2003;13:2498-2504.

17 Maere S, Heymans K, Kuiper M: BiNGO: a Cytoscape plugin to assess overrepresentation of gene ontology categories in biological networks. Bioinformatics 2005;21:3448-3449.

18 Lamb J, Crawford ED, Peck D, Modell JW, Blat IC, Wrobel MJ, Lerner J, Brunet JP, Subramanian A, Ross KN, Reich M, Hieronymus H, Wei G, Armstrong SA, Haggarty SJ, Clemons PA, Wei R, Carr SA, Lander ES, Golub TR: The Connectivity Map: using gene-expression signatures to connect small molecules, genes, and disease. Science 2006;313:1929-1935. 


\section{Kidney \\ Blood Pressure Research}

19 D'Amico G: The commonest glomerulonephritis in the world: IgA nephropathy. Q J Med 1987;64:709-727.

20 Gharavi AG, Yan Y, Scolari F, Schena FP, Frasca GM, Ghiggeri GM, Cooper K, Amoroso A, Viola BF, Battini G: IgA nephropathy, the most common cause of glomerulonephritis, is linked to 6q22-23. Nat Genet 2000;26:354-357.

-21 Ju W, Eichinger F, Bitzer M, Oh J, Mcweeney S, Berthier CC, Shedden K, Cohen CD, Henger A, Krick S: Renal gene and protein expression signatures for prediction of kidney disease progression. Am J Pathol 2009;174:2073-2085.

22 Cohen CD, Frach K, Schlöndorff D, Kretzler M: Quantitative gene expression analysis in renal biopsies: a novel protocol for a high-throughput multicenter application. Kidney Int 2002;61:133-140.

-23 Bell G, Gordon A, Lee P, Doig A, Macdonald M, Thomson D, Anderton J, Robson J: Proliferative glomerulonephritis and exposure to organic solvents. Nephron 1985;40:161-165.

24 Porro A, Lomonte C, Coratelli P, Passavanti G, Ferri GM, Assennato G: Chronic glomerulonephritis and exposure to solvents: a case-referent study. Br J Ind Med 1992;49:738-742.

25 Mercado C, Jaimes EA: Cigarette smoking as a risk factor for atherosclerosis and renal disease: novel pathogenic insights. Cur Hypertens Rep 2007;9:66-72.

26 Jaimes EA, Tian R-X, Raij L: Nicotine: the link between cigarette smoking and the progression of renal injury? Am J Physiol Heart Circ Physiol 2007;292:H76-H82.

27 Jain G, Jaimes EA: Nicotine signaling and progression of chronic kidney disease in smokers. Biochem Pharmacol 2013;86:1215-1223.

28 Bronchud M, Howell A, Crowther D, Hopwood P, Souza L, Dexter T: The use of granulocyte colonystimulating factor to increase the intensity of treatment with doxorubicin in patients with advanced breast and ovarian cancer. Br J Cancer 1989;60:121-125.

-29 Von Der Maase H, Hansen S, Roberts J, Dogliotti L, Oliver T, Moore M, Bodrogi I, Albers P, Knuth A, Lippert C: Gemcitabine and cisplatin versus methotrexate, vinblastine, doxorubicin, and cisplatin in advanced or metastatic bladder cancer: results of a large, randomized, multinational, multicenter, phase III study. J Clin Oncol 2000;18:3068-3077.

-30 Aas T, Børresen A-L, Geisler S, Smith-Sørensen B, Johnsen H, Varhaug JE, Akslen LA, Lønning PE: Specific P53 mutations are associated with de novo resistance to doxorubicin in breast cancer patients. Nat Med 1996;2:811-814

31 Von Pawel J, Schiller JH, Shepherd FA, Fields SZ, Kleisbauer J, Chrysson NG, Stewart DJ, Clark PI, Palmer MC, Depierre A: Topotecan versus cyclophosphamide, doxorubicin, and vincristine for the treatment of recurrent small-cell lung cancer. J Clin Oncol 1999;17:658-658.

-32 Singal PK, Siveski-Iliskovic N, Hill M, Thomas TP, Li T: Combination therapy with probucol prevents adriamycin-induced cardiomyopathy. J Mol Cell Cardiol 1995;27:1055-1063.

33 Hahn H, Park YS, Ha IS, Cheong HI, Choi Y: Age-related differences in Adriamycin-induced nephropathy. Pediatr Nephrol 2004;19:761-766.

-34 Malarkodi K, Varalakshmi P: Lipoic acid as a rescue agent for adriamycin-induced hyperlipidemic nephropathy in rats. Nutr Res 2003;23:539-548.

-35 Rogers TB, Inesi G, Wade R, Lederer W: Use of thapsigargin to study Ca2+ homeostasis in cardiac cells. Biosci Rep 1995;15:341-349.

-36 Hakii H, Fujiki H, Suganuma M, Nakayasu M, Tahira T, Sugimura T, Scheuer P, Christensen S: Thapsigargin, a histamine secretagogue, is a non-12-0-tetradecanolphorbol-13-acetate (TPA) type tumor promoter in twostage mouse skin carcinogenesis. J Cancer Res Clin Oncol 1986;111:177-181.

-37 Thastrup 0, Cullen PJ, Drøbak B, Hanley MR, Dawson AP: Thapsigargin, a tumor promoter, discharges intracellular Ca2+ stores by specific inhibition of the endoplasmic reticulum Ca2 (+)-ATPase. Proc Natl Acad Sci USA 1990;87:2466-2470.

-38 Dombroski BA, Nayak RR, Ewens KG, Ankener W, Cheung VG, Spielman RS: Gene expression and genetic variation in response to endoplasmic reticulum stress in human cells. Am J Hum Genet 2010;86:719-729.

39 Sinha R, Shekhar H, Sharma N, Titiyal JS, Vajpayee RB: Posterior capsular opacification: A review. Indian J Ophthalmol 2013;61:371. 